\title{
Analysis of Various Kids Search Engines
}

Deepshikha Patel ${ }^{1 *}$, Prashant Kumar Singh ${ }^{2}$

1. Dr. K. N. Modi University,

2. ISC Software Pvt Ltd,

*Corresponding author: Deepshikha Patel

Dr. K. N. Modi University,

Newai, Rajasthan, India.

E-mail: 23.deepshikha@gmail.com

Received: December 30, 2013, Accepted: January 6, 2014, Published: January 8, 2014.

\section{ABSTRACT}

Web is the most commonly used resource type for finding information these days. Most search engines results are not kids oriented that's why there are several search engines available in the market to fulfill the need of kids. In this paper we will analyze various search engines on different points like design, interactivity, ease of access, technology used, graphics etc.

Keywords: Search Engines; Web Search Engines; Meta Search Engines; Searching; Kids, Kids Search.

\section{INTRODUCTION}

Kids are active information seeker these days. The age of surfing the web is decreasing day by day. Kids use internet for their school project work, playing games, watching cartoons etc. World Wide Web is enormous in size and it contains lots of information that may not be appropriate for the kids. To protect kids from this inappropriate information need of search engines specific to kids arose. Apart from harmful content there are lots of other issues for developing search engines for kids like access, query formation, search result selection, readability etc. First of all interface of search engines must be interactive including graphics and various colors as kids are attracted towards colors. Kids feel difficulty in formation of query for finding exact information. There must be automatic query suggestion as per kid's requirements. Kids also face problem while selecting from search engine result as millions of pages are shown against the query string. So the indexing should be proper as per kids. It is good to see few but relevant web pages in the search than thousands of irrelevant pages. Kids are also facing readability issues on the web because most of the web pages are designed are adult oriented. Kids less vocabulary power is major issue as many of them unable to understand the content found. So as we have discussed various issues why the kid specific search engines are required, we will discuss them in the next section.

\section{KIDS SEARCH ENGINES}

There are various search engines designed primarily to serve the needs of kids. In this section we will discuss most of them one by one with the features provided and problems with them.

\section{Boolify}

Website: http://www.boolify.com/

This is based on Google's customized search. Boolify[1] helps children to bridge the gap by visualizing the process and letting them interact with the concept behind the search process in a hand-on way. Boolify's primary audience is elementary and middle schoolchildren.

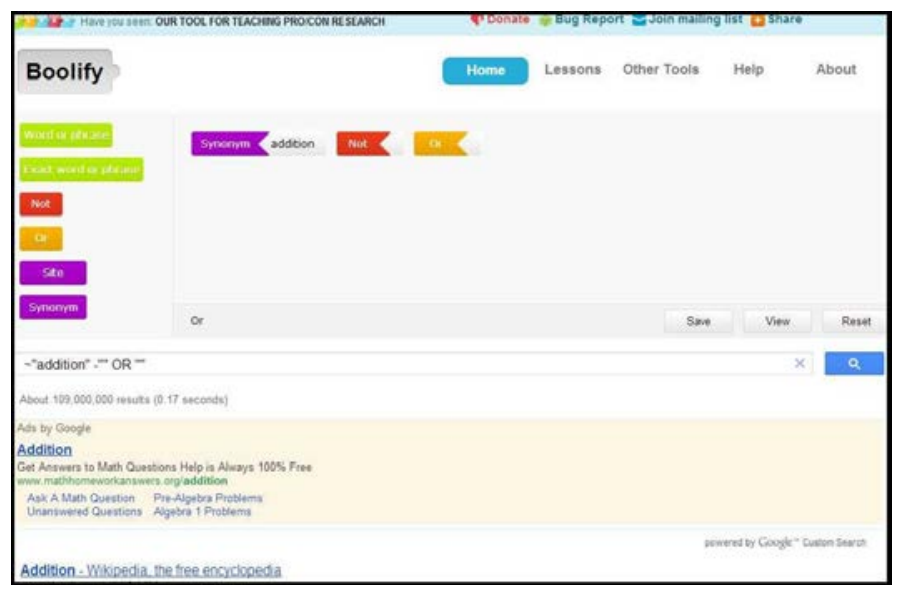

\section{Quintura}

Website: http://quinturakids.com/

Quintura for Kids [2] is powered by Yahoo. It offers a slightly different way of searching as compared to other sites. It is a more visual search engine using a keyword cloud for easy navigation. You start off your search with a keyword in the text box and then modify it with any of the keywords in the cloud. 
Quintura displays five results per page. You may miss it, but clicking on the surrounding icons takes you to the five preset search categories “ Music, History, Animals, Sports and recreation, and Games.

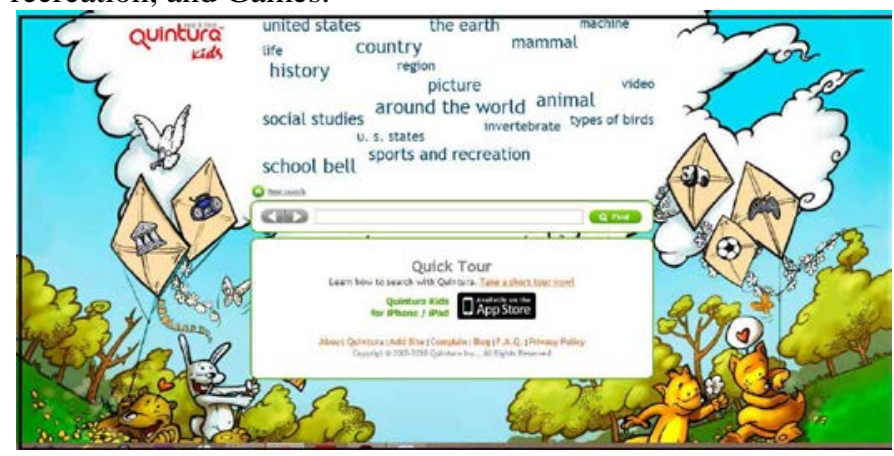

Quintura Kids is the first visual mobile search engine for children. Its intuitive interface makes web search real fun! Along with a common search box, Quintura Kids features a dynamic tag cloud changing depending on your preferences. Surfing with Quintura Kids is easy and kid-friendly even for the youngest. Just tap the words in the tag cloud! The cloud dynamically changes suggesting you with most popular concepts we pick up from the relevant web pages. These help you define the best direction for further search and refine your request. Search is only within manually picked up kid-related web sites. No results you get contain adult content. So parents and teachers can feel safe and you can freely recommend our application to your schoolchildren and their parents.

\section{KidRex}

Website: http://www.kidrex.org/

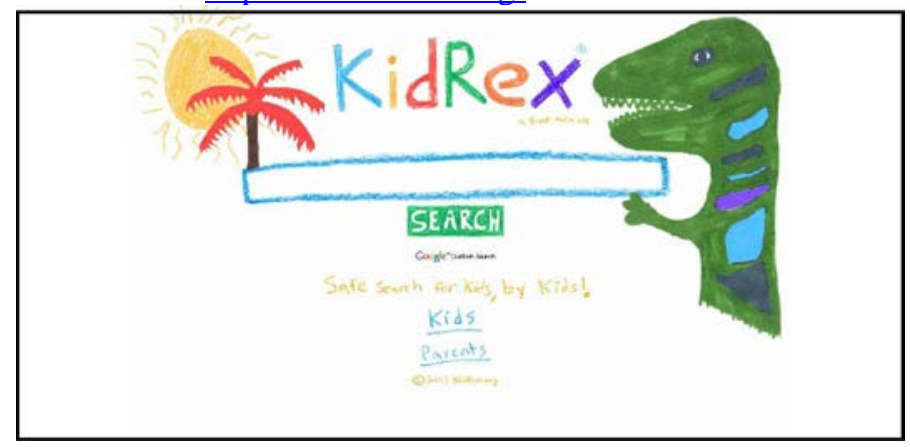

KidRex[3] is a custom Google search engine for kids launched in December 2008. The interface is just like a child's crayon drawing (the dinosaur stands guard). The site also has a colorful hand-drawn crayon and colored marker design. It uses SafeSearch and tries to keep all the results as antiseptic as possible.In addition to Google's SafeSearch, KidRex also has its own database of inappropriate websites and keywords which further help to keep the results clean.

KidRex is listed in top50 websites 2013 by TIME.

KidRex also has website removal tool if parents find any inappropriate.

\section{KidzSearch}

Website: http://www.kidzsearch.com/
KidzSearch [4] is Based on Google's Custom Seacrch. Kidzsearch has query suggestion to help kids finding appropriate information.

Disadvantage: Ads

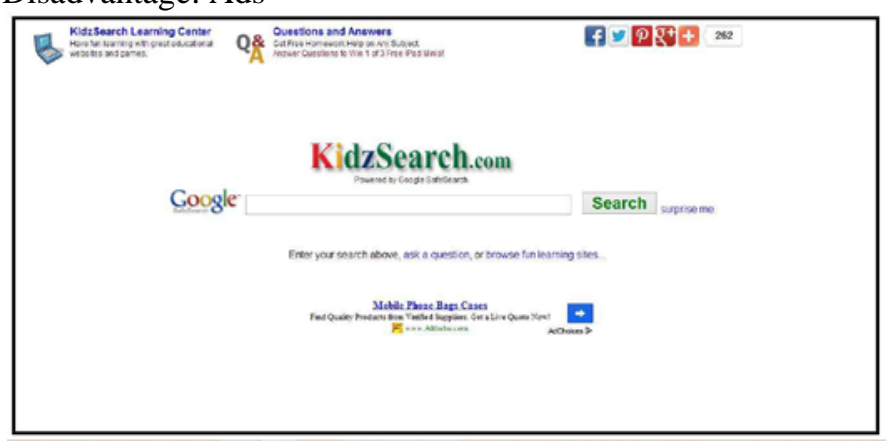

\section{SquirrelNet}

Website:

http://www.squirrelnet.com/search/Google_SafeSearch.asp

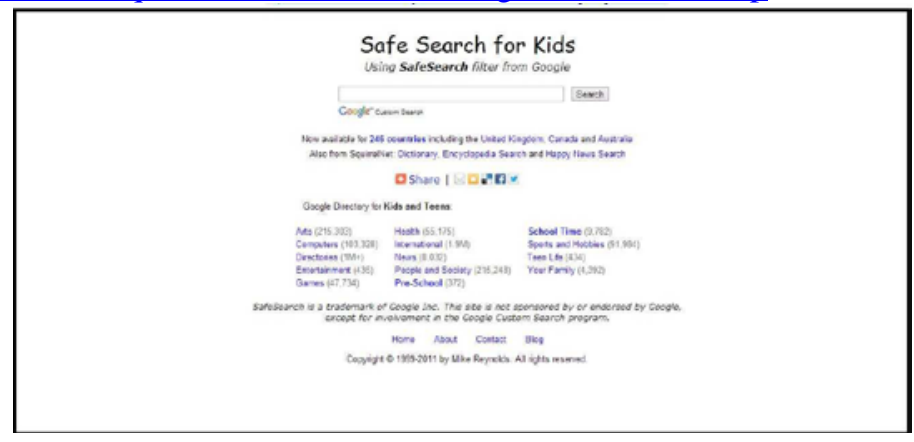

SquirrelNet [5] is not a kids only search engine. SquirrelNet for kids is a part of this search engines that has Google SafeSearch activated. From the homepage itself, you can also access the Google directory of websites relevant for children.

Problem: The problem with Squirrelnet Google Safe search for kids is that you need to continue providing access to the domain www.google.com in order for it to work, since it uses java scripts API that require access to files from that domain. That means that regular, non restricted Google searches are still possible.

Search text box also suggest previous search performed in the browser, which is not safe for kids.

\section{Safe Search Kids}

Website: http://www.safesearchkids.com/

Safe Search Kids [6] is based on Google’s custom Search. Search text box also suggest previous search performed in the browser. This search engine is showing the basic search results from the Google.

It has the same problem as discussed for SquirrelNet. Hence not safe for kids. 


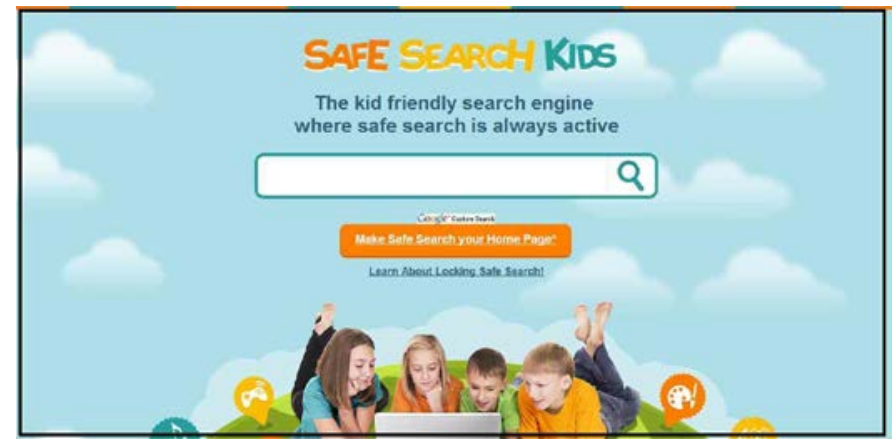

Aga-Kids

Website: http://aga-kids.com/

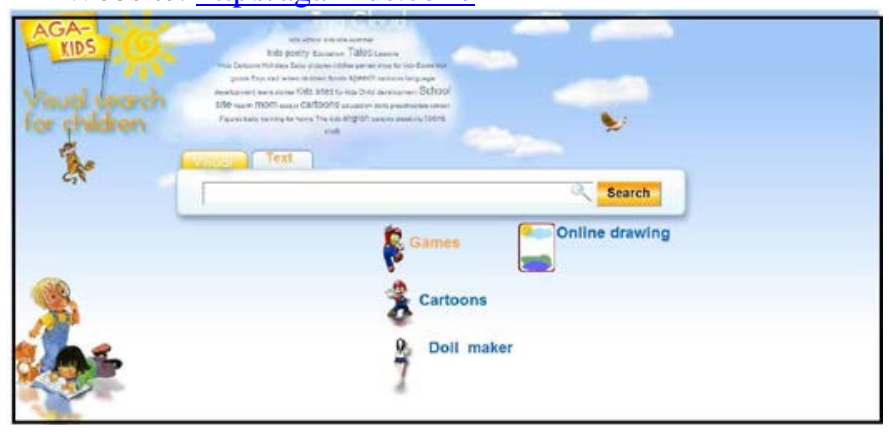

Aga-Kids [7] is a visual search engine for kids and one of the more colorful ones you will see. You can choose between a visual search and a text search. The search results come up as interactive and animated thumbnails.

The searching system "AGAKIDS" is created to help kids and children to find attractive resources in the wide fields of Internet.

The search results may be limited because the search engine searches only websites that are made for children.

\section{Dib Dab Dooand Dilly too}

Website: http://www.dibdabdoo.com/

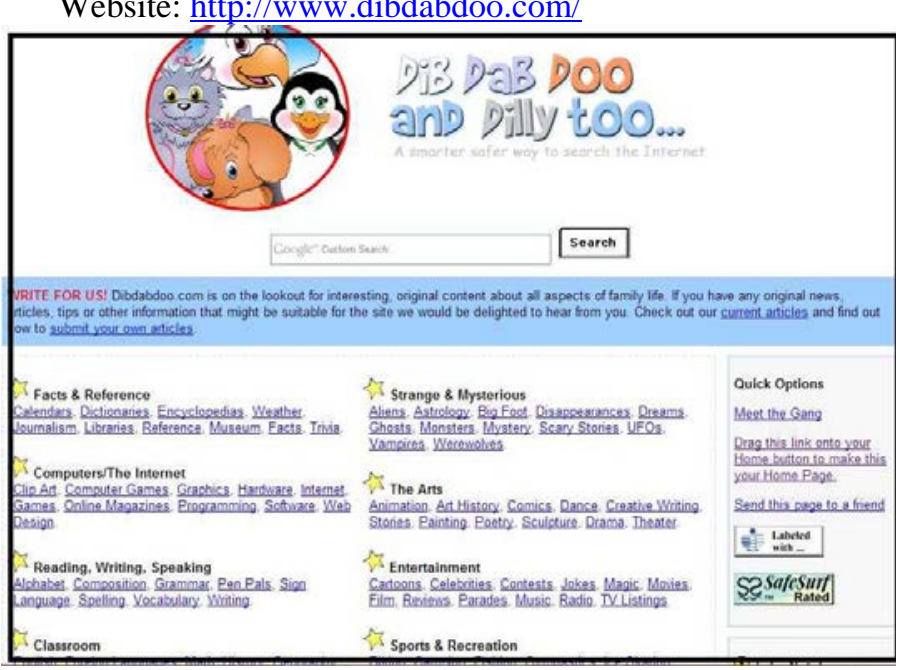

If any name shouts out that it's a search engine for kids, then this is it. The search engine is again based on Google Custom Search and it tries to keep the content as children friendly as possible. Custom search helps to keep out a lot of unsavory links, but it is definitely not foolproof. Most of the search engines for kids also display ads with some undesirable ones sneaking in. Parental control software in combination with these search engines can help to keep children shielded from the bad side of the web. It is a tough battle but parents can worry a little less. These ten search engines for kids are just the search tools for some unattended browsing around an unsafe web.[8]

Search text box also suggest previous search performed in the browser.

$$
\text { Disadvantage: Ad }
$$

\section{KidsClick!}

Website: http://www.kidsclick.org/

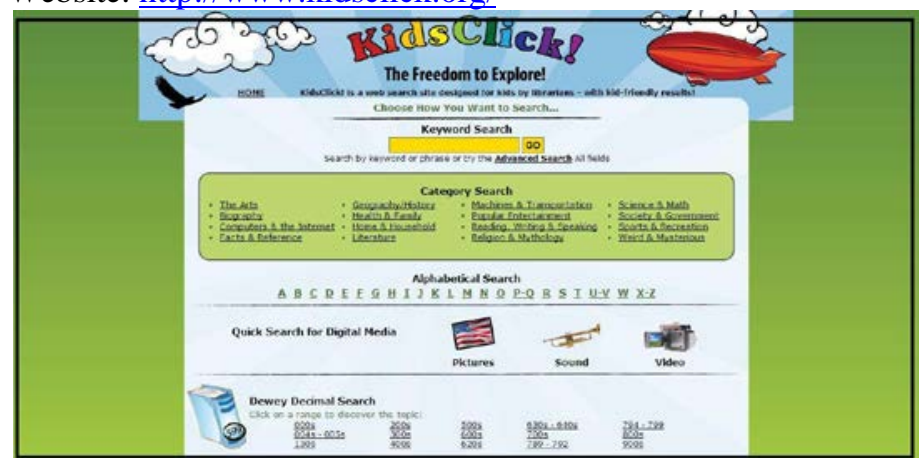

Backed by librarians, KidsClick lists about 5,000 web sites in various categories.

KidsClick [9] makes it clear in its About page that it is not an internet filter. It is a directory of good resources (a 600+ strong subject list) which kids can use for information or schoolwork. KidsClick is owned and run by the School of Library and Information Science (SLIS) at Kent State University. As the web resource links to a comprehensive collection of good, clean sites, the KidsClick interface is without any ads.

\section{SweetSearch}

Website: http://www.sweetsearch.com

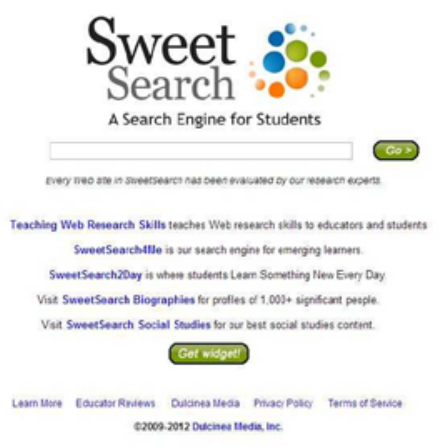

SweetSearch [10] features the highest quality websites created for kids. It does not rely on filter, but rather is a white list of approved sites that are searched by Google's technology.

\section{Looksmart's KidsDirectory}

Website: http:/www.looksmart.com 


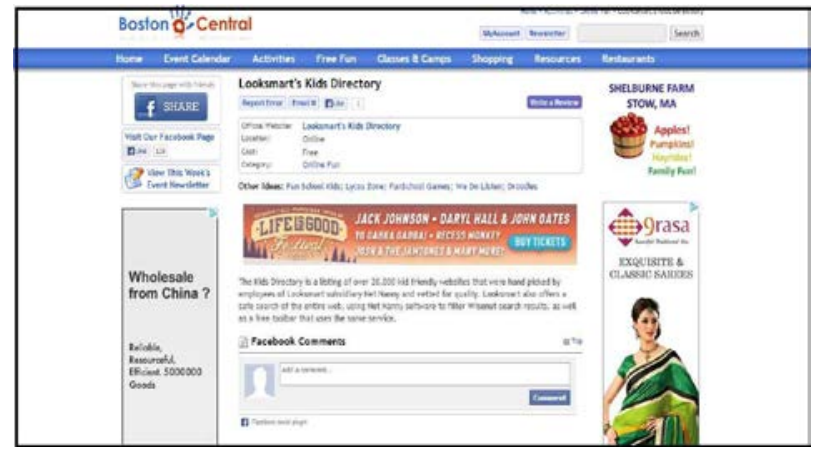

The Kids Directory[11] is a listing of over 20,000 kid friendly websites that were hand picked by employees of Looksmart subsidiary Net Nanny and vetted for quality. Looksmart also offers a safe search of the entire web, using Net Nanny software to filter Wisenut search results, as well as afree toolbar that uses the same service.

Look Smart has never accepted adult content for listing within its directory results. However, obscure queries might bring these up in the crawler-based results that are sometimes provided.

\section{Awesome Library}

Website: http://www.awesomelibrary.org/

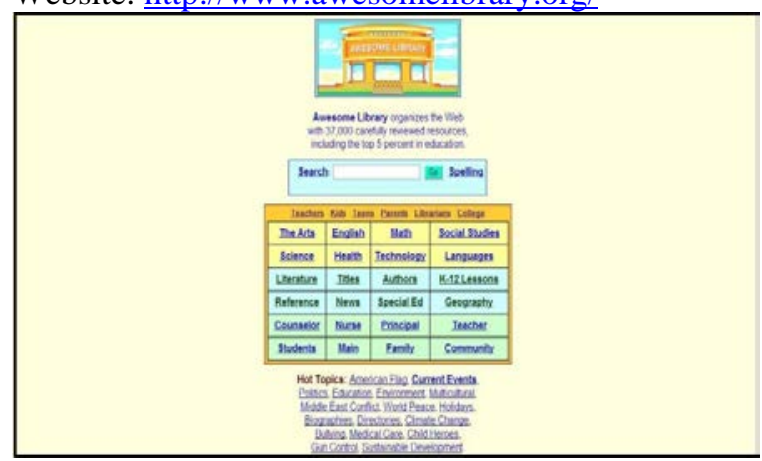

Awesome Library[12] has over 14,000 sites classified into a directory, specifically organized for teachers, students and parents. Information can be found by browsing or searching.

Problems :Ads

\section{FactMonster}

Website: http://www.factmonster.com/

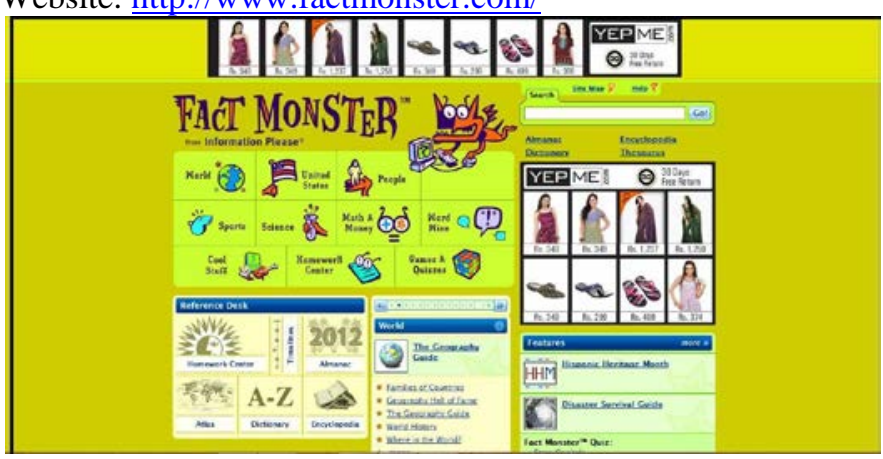

Fact Monster[13] provides facts and information oriented around the needs of children.
A good, comprehensive site offering a "history of the lunch box" when asked for Spidey-related info along with biographies of the character's creator. Laura Ingalls Wilder's name brought up the basic information on her life along with links to "places where women made history." Provided many links to famous Georges along with the one requested. Most of the results appeared to come from encyclopedia articles.

Problem: Ads

\section{KidsGov}

Website: http://www.kids.gov/

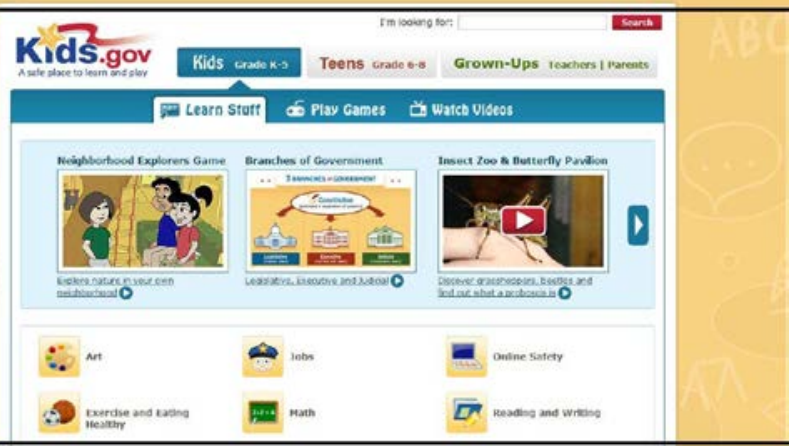

From the U.S. Federal Citizen Information Center, this directory provides links to government-related kids' sites along with some of the best kids' sites from other organizations, grouped by subject. Search results are based on Bing. [14]

Feature: query suggestion, Ads free

\section{Cybersleuth Kids}

Website: http://cybersleuth-kids.com/

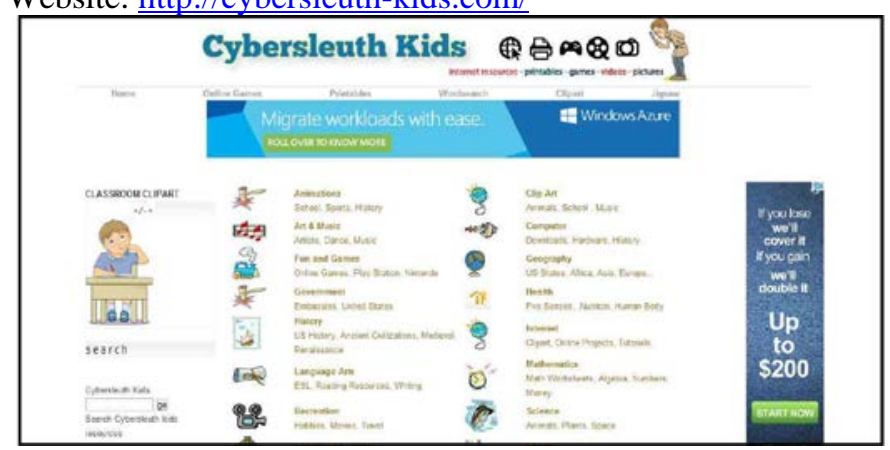

Touts itself as an Internet guide for kids ages kindergarten through grade 12. Provided no results for Spiderman and few for Laura Ingalls Wilder. Led to web site focused on another favorite female heroine: Nancy Drew. George Washington pulled up info on the first president from academic kids.com and as well as info on another famous George: George Washington Carver.[15]

Problem: Ads

\section{Yahoo Kids!}

Website: www.kids.yahoo.com (Currently unavailable)

Yahoo Kids[16] was established in 1996 by Yahoo to give children a platform to find appropriate, safe internet content. It was known as Yahooligans till November 2006. Yahoo for kids designed for ages 7 to 12 . Sites are hand-picked to be appropriate for children. Also, unlike normal Yahoo, searches will not bring back matched found by crawling the web, if there is no match from within the Yahooligan listings. This prevents possibly objectionable sites from slipping onto the screen. 
Additionally, adult-oriented banner advertising will not appear within the service.

Yahoo Kids was the oldest online search directory for kids to find age appropriate information. The website has been used for Education and Entertainment purposes as well. Yahoo Kids is the doorway to Yahoo's Directory of websites and URLs exclusively for kids. The home page is colorful, engaging and full of cool stuff to keep kids engaged.

Yahoo Kids closed on April 30, 2013 so the company could redirect its resources to mobile applications.

\section{Ask Kids}

Ask Kids is a search engine for kids from Ask.com's pool of web resources. The search page resembles a school note book. Apart from the search box, five preset search categories Schoolhouse, Movies, Games, Videos and Images, help out the kiddies research all kinds of stuff.

Kids can jump from the search results to images, narrow or expand the search, find related names and other information. It borrows the features from Ask.com and its regular search, but keeps it simple for kids.

Ask Kids is not available now.

\section{Ask Jeeves}

Website: http://www.ajkids.com/

Ask Jeeves is a unique service where you enter a question, and Ask Jeeves tries to point you to the right web page that provides an answer. At Ask Jeeves For Kids, answers have been vetted for appropriateness. Also, if Ask Jeeves cannot answer a question, it pulls results from various search engines in its metacrawler mode. At Ask Jeeves For Kids, no site that is on the CyberPatrol block list is supposed to be listed.

\section{ANALYSIS \\ In the previous section we have seen popular search engines for kids. In this section we will discuss features and problems associated with Kids search engines. \\ After analyzing so many kids search engines we find good features that are very helpful and safe for kids searching process. On the other hand we have also seen some problems associates with some of the search engines which may redirect kids to other harmful or inappropriate websites. \\ Here we will focus on features and problems we have analyzed.}

\section{FEATURES}

\section{Query Suggestion}

Query suggestion is a very good features found in some of search engines discussed above because it is very hard to write appropriate keyword against the information they are looking for.

\section{Ads Free Search}

Ads free search is very import while the search is concerned with Kids. Many search engines are not providing Ads free services to Kids but some of them are Ads free search engines. Google's Customized Ads may redirect kids to another place or can divert their mind.

\section{Problems}

\section{Cached Query Suggestion}

Many of the search engines are showing the previously searched keywords from the browser's cache. These keywords may be of any kind not appropriate for kids.

\section{Basic Search results}

Some of the search engines discussed above are using Google's safe search but showing basic search results from the Google.

\section{Customized Ads}

Customized ads may not be appropriate for kids. There is no use of including ads in the Kids Search engines. Some search engines discussed above are not supporting Ads free search.

\section{CONCLUSION}

In the Internet it is very difficult to find the specific and useful kids oriented sites as there are enormous sites with the content not fitted for the kids interests, mentality and demands. For this purpose there are many search engines designed to provide easy and safe services for kids.

In this paper we have discussed most popular search engines primarily designed for kids. We have also seen the features and problems associated with them. There is possibility to improve these search engines to provide better services to kids.

\section{REFERENCES}

1. Boolify, http://www.boolify.com/

2. Quintura, http://quinturakids.com/

3. KidRex, http://www.kidrex.org/

4. KidzSearch, http://www.kidzsearch.com/

5. SquirrelNet, http://www.squirrelnet.com/search/Google_SafeSear ch.asp

6. Safe Search Kids, http://www.safesearchkids.com/

7. Aga-Kids, http://aga-kids.com/

8. Dib Dab Dooand Dilly too, http://www.dibdabdoo.com/

9. KidsClick! , http://www.kidsclick.org/

10. SweetSearch, http://www.sweetsearch.com

11. Looksmart's KidsDirectory, http://www.looksmart.com/

12. Awesome Library, http://www.awesomelibrary.org/

13. Fact Monster, http://www.factmonster.com/

14. KidsGov, http://www.kids.gov/

15. Cybersleuth Kids, http://cybersleuth-kids.com/

16. Yahoo Kids!,

Citation: Deepshikha Patel (2014) Analysis of Various Kids Search Engines. J. of Computation in Biosciences and Engineering. V1I1. 